\title{
Intravenous low-grade endometrial stromal sarcoma with intracardiac extension: A case of inaccurate tumor location on contrast-enhanced computed tomography
}

\author{
YUYA NOGAMI $^{1}$, WATARU YAMAGAMI ${ }^{1}$, JUNKO MAKI $^{1}$, KOUJI BANNO $^{1}$, NOBUYUKI SUSUMU $^{1}$, \\ KOICHI TOMITA ${ }^{2}$, KENTARO MATSUBARA ${ }^{2}$, HIDEAKI OBARA $^{2}$, YUKO KITAGAWA $^{2}$ and DAISUKE AOKI ${ }^{1}$ \\ Departments of ${ }^{1}$ Obstetrics and Gynecology, and ${ }^{2}$ Surgery, Keio University School of Medicine, Tokyo 1608582, Japan
}

Received June 2, 2015; Accepted November 17, 2015

DOI: $10.3892 / \operatorname{mco} .2015 .691$

\begin{abstract}
We herein report a case of low-grade endometrial stromal sarcoma with intracardiac extension in a 58-year-old woman with a uterine tumor with intravascular involvement. The tumor was suspected preoperatively to be an endometrial stromal sarcoma by magnetic resonance imaging. The extent of intravascular involvement was determined to be below the level of the renal veins on preoperative contrast-enhanced computed tomography (CT). However, perioperative echography revealed that the tumor extended to the right atrium. An emergency cardiotomy with extracorporeal circulation was required. The risk of tumor embolism was reduced through transection of the inferior vena cava, but the tumor was difficult to remove completely. Postoperative hormonal therapy reduced the size of the residual tumor and no recurrence was detected for 1 year. The preoperative contrast-enhanced CT was unable to detect the free-floating intravascular tumor. This case illustrates a limitation of CT and indicates that accurate determination of the tumor extent for planning a surgical strategy in similar cases should be performed using multiple imaging methods.
\end{abstract}

\section{Introduction}

Uterine sarcoma is a relatively rare tumor that accounts for $\sim 8 \%$ of all uterine malignancies (1). In the Japanese population, $13-19 \%$ of these tumors are classified as endometrial stromal sarcoma (ESS) $(2,3)$. Due to the changes in the World Health Organization classification in 2003, which classified high-grade ESS as undifferentiated endometrial sarcoma (4), low-grade ESS (LG-ESS) may simply be referred to as ESS in some cases, although the classification reverted to its original

Correspondence to: Dr Wataru Yamagami, Department of Obstetrics and Gynecology, Keio University School of Medicine, 35 Shinanomachi, Shinjuku-ku, Tokyo 1608582, Japan

E-mail: gami@z8.keio.jp

Key words: endometrial stromal sarcoma, contrast-enhanced computed tomography, tumor thrombosis, intravascular leiomyomatosis, intracardiac extension form in 2014 (5). LG-ESS presents with lymph node metastasis in $9-33 \%$ cases $(6,7)$ and displays characteristics that include frequent development as an intravascular tumor with intracardiac extension (ICE). The number of reported cases of LG-ESS with clinically obvious intravascular extension was only 19 over the last 38 years $(8,9)$; however, up to one-third of the cases presented with extrauterine extension, including microscopic worm-like plugs within the vessels (4). Such tumors must be differentiated from deep venous thrombosis, whereas benign leiomyoma presents with characteristics similar to intravenous leiomyomatosis. Other uterine malignancies may also rarely present as a tumor embolism (10).

An intravascular tumor is associated with a direct risk of sudden death due to pulmonary embolism; thus, it is crucial to plan a precise surgical strategy based on the exact extent of the intravascular tumor. We treated a case of LG-ESS with ICE, in which preoperative contrast-enhanced computed tomography (CT) indicated a misleading tumor location. We herein describe this case for educational purposes and provide a literature review on the imaging modalities.

Written informed consent was obtained from the patient for the publication of her medical details.

\section{Case report}

The patient was a 58-year-old woman who was gravida 5, para 3 and postmenopausal. A fibroid in her uterus had been diagnosed at 52 years of age, but no medical intervention was performed due to the imminent menopause. The patient had no other medical history and had undergone no gynecological follow-up. During a routine medical checkup, a marginal elevation of the serum liver enzymes and impaired glucose tolerance were found, but the patient was asymptomatic.

The patient visited a general hospital for an investigation of the elevated liver enzymes. A thoracoabdominal CT revealed an irregular tumor in the uterus, with intravenous spread of the tumor to the inferior vena cava (IVC). On pelvic magnetic resonance imaging (MRI), an irregular tumor was identified in the right posterior wall of the uterus. The tumor mainly exhibited low-signal intensity on T1-weighted imaging (T1WI), mainly high-signal intensity on T2WI (Fig. 1), a mild to intermediate effect with gadolinium contrast agent, and a 

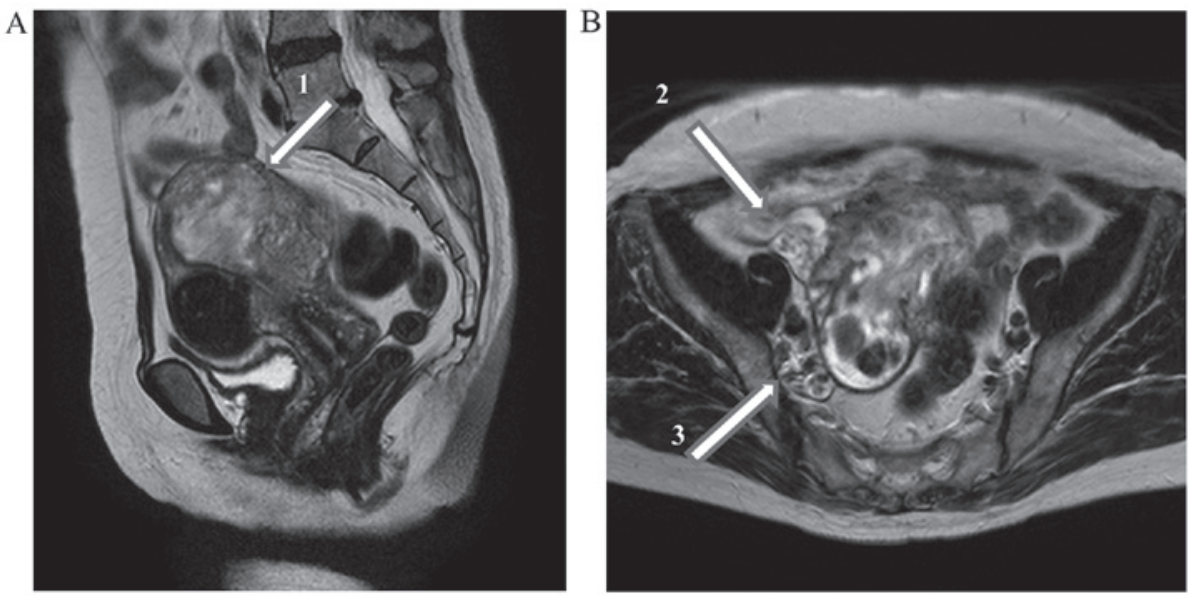

Figure 1. T2-weighted imaging in preoperative pelvic magnetic resonance imaging. (A) Sagittal slice showing an irregular tumor with high-signal intensity extending from the cavity to the right posterior wall of the uterus (arrow 1). (B) Axial slice showing extension of the tumor to the right adnexa and vessels (arrow 2) and the right internal iliac vein (arrow 3).

high-signal intensity on diffusion weighted imaging (DWI). The presence of a non-endometrial tumor located near the cavity suggested that sarcoma had invaded from just below the endometrium, with a low density on T2WI due to the normal myometrium underlying the tumor invasion. These findings were compatible with a diagnosis of ESS.

The patient was admitted to our hospital for treatment of the uterine tumor. Following emergency admission, the patient underwent dynamic contrast-enhanced $\mathrm{CT}$ of the whole body to determine the extent of the tumor. CT in the venous phase indicated that the tip of the intravenous tumor was at the level of the renal veins (Fig. 2), without thrombosis or a tumor pulmonary embolus. The routes of extension were via the internal iliac and ovarian veins. The MRI scan indicated that the tumor was located near the cavity, but the cytological examination of the endocervix and endometrium was negative for malignancy.

A surgical team of gynecological oncologists and vascular surgeons was assembled. Anticoagulation with unfractionated heparin was performed until surgery to prevent thrombogenesis. Considering the extent of the tumor, it was feared that insertion of an IVC filter would interfere with the manipulation of the liver. Thus, a filter for prevention of pulmonary embolism was not inserted preoperatively. Laparotomy was performed for hysterectomy, bilateral salpingo-oophorectomy and debulking of the intravenous tumor. The gynecological oncologist team performed a hysterectomy with extension of the range of resection to that of radical hysterectomy on the right side of the uterus, in which small vessels were invaded by the tumor. However, it was difficult to remove the tumor completely from the deep parametrium and paracolpium. A perioperative pathological examination by frozen section analysis did not indicate obvious malignancy. Thus, we did not perform a complete resection, considering the balance between the invasiveness of the operation and the therapeutic merit.

Subsequently, the vascular surgery team took over to remove the intravascular tumor. On preoperative planning, the tumor was present below the level of the renal veins; however, a perioperative ultrasound scanning of the IVC revealed a free-floating tumor in the form of a thin multinodular plate extending to the right atrium (Fig. 3). Manipulation of the liver was required, but

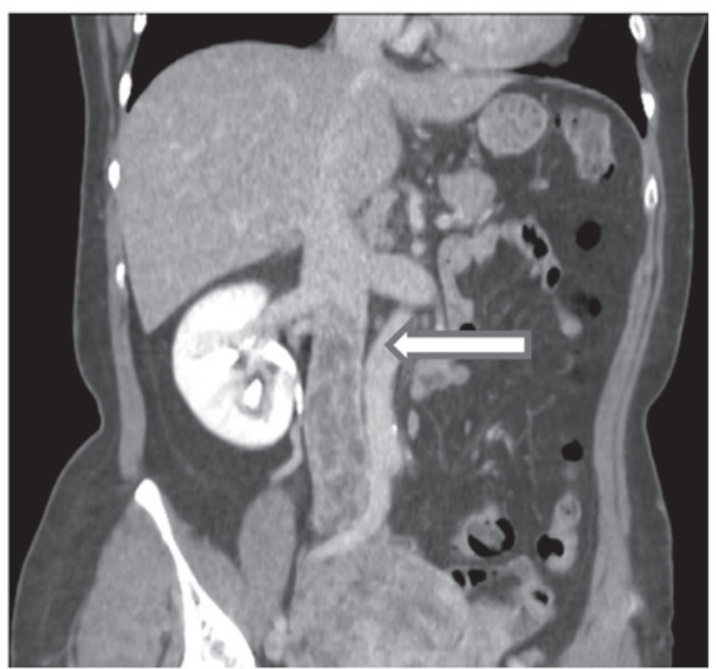

Figure 2. Preoperative abdominal contrast-enhanced CT. Coronal slice showing an irregular filling defect due to an intravascular tumor in the inferior vena cava below the level of the renal vein (arrow).

was associated with the risk of tumor pulmonary embolisation. A cardiovascular surgery team performed emergency thoracotomy with median sternotomy and removed the intracardiac tumor through cardiotomy of the right atrium using extracorporeal circulation without circulatory arrest. After closing the heart, a radiologist inserted a filter in the IVC immediately below the right atrium and the vascular surgery team exposed the IVC behind the liver, cut the vessel wall open and removed the intravascular tumor under IVC cross-clumping (Fig. 4).

Following removal of the tumor above the renal veins, the gonadal veins were separated from the IVC, and the IVC was detached below the level of the renal veins. This procedure was performed to prevent future tumor pulmonary embolisation, as the tumor was not removed completely. The amount of perioperative blood loss was 7,200 ml. Transfusion of 38 units of packed red blood cells, 30 units of frozen fresh plasma and 10 units of platelets was required.

On transecting the IVC, bilateral grade 2 pedal edema appeared immediately after the procedure, but had improved 

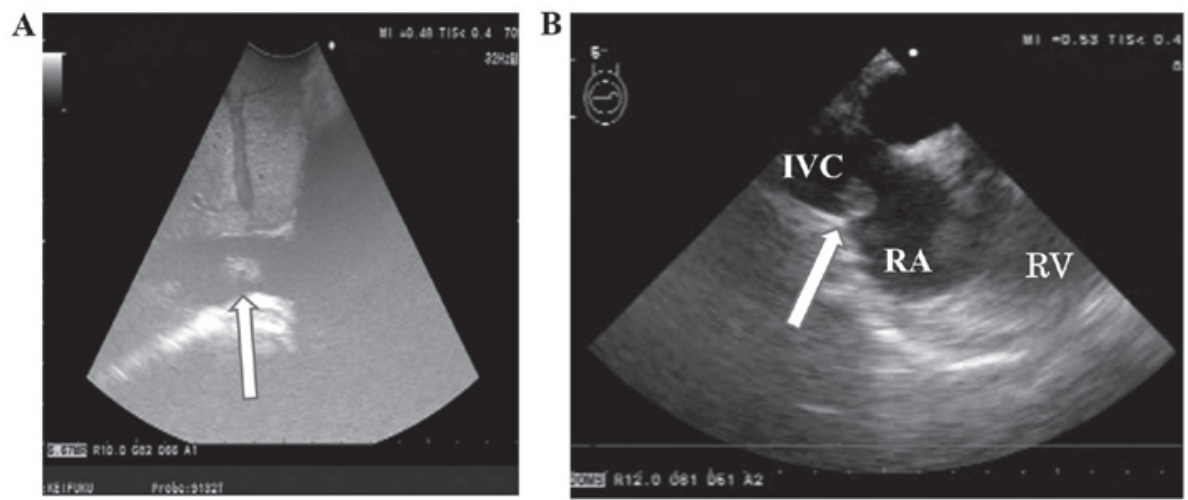

Figure 3. Perioperative ultrasound scan of the inferior vena cava. (A) Transabdominal echogram showing a free-floating tumor in the IVC behind the liver. (B) Transesophageal echogram showing the tip of the intravascular tumor in the right atrium. IVC, inferior vena cava; RA, right atrium; RV, right ventricle.

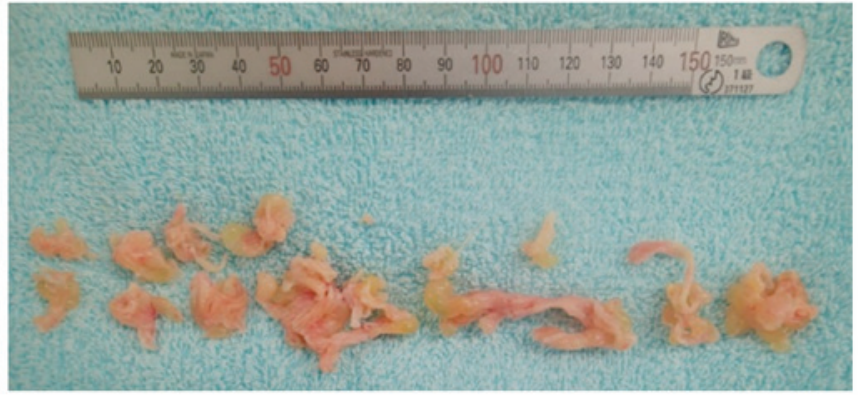

Figure 4. Intravascular tumors removed from the inferior vena cava above the level of the renal veins. These tumors were not detected by contrast-enhanced computed tomography, possibly due to their flat plate-like shape and their free-floating and mobile nature.

by postoperative day (POD) 10. Contrast-enhanced CT on POD 10 revealed a thrombus in the bilateral common to internal iliac veins. The thrombus was originally right-sided and had an extensive range. It was difficult to distinguish between the thrombus and tumor growth; thus, anticoagulation therapy with warfarin was started for differentiation. No other adverse events occurred, including surgical site infection, and the patient was discharged on POD 23.

Pathological examination of the resected uterine tumor established a final diagnosis of LG-ESS. Benign leiomyoma also existed in the uterine corpus, but the intravascular tumors were consistent with sarcoma. Thus, the tumor was classed as clinical stage IIIA (International Federation of Gynecology and Obstetrics 2008). Residual tumors were present in the right common iliac vein, internal iliac vein, parametrium, and paracolpium. Therefore, MRI with a gadolinium contrast agent was performed 1 month after the operation. The range of the poor-contrast area in the bilateral common to internal iliac veins was reduced by warfarin, compared with that on enhanced CT immediately after the operation. The area in which the original intravascular residual tumor displayed an irregular pattern in T1WI, T2WI and DWI was not extended. Thus, we concluded that the tumor had not undergone a sudden increase in size.

Postoperative hormonal therapy was recommended and the patient received $400 \mathrm{mg} / \mathrm{day}$ medroxyprogesterone acetate (MPA) for a year. The residual tumor almost disappeared and the patient remains recurrence-free for 1 year following MPA therapy.

\section{Discussion}

We treated a case of uterine tumor with intravascular involvement. The imaging findings indicated a straightforward diagnosis of sarcoma with tumor thrombus; however, benign leiomyoma presents similarly to intravenous leiomyomatosis. A diagnosis of malignancy based on intravenous tumor development detected by imaging may lead to unnecessary chemotherapy or radiotherapy (11). MRI is a useful tool for distinguishing between benign leiomyoma and sarcoma, as in the present case, but degenerated benign leiomyomas may have findings similar to those of sarcomas, which may present with difficulties in differentiating between the two $(12,13)$. As pathological examination of stromal tumors is difficult to perform preoperatively, it is crucial to diagnose by surgical resection. In cases with intravascular lesions, it is also crucial to prevent future pulmonary embolism. Thus, the principle of treating such cases is to plan an optimal surgical strategy.

In planning the surgical strategy, it is important to be able to accurately predict the tumor extent preoperatively; however, this proved not to be possible in the current case. On preoperative planning, the tip of the tumor was considered to be located at the level of the renal veins, based on contrast-enhanced CT. Intravascular tumors located in the IVC above the renal veins were not identified on this scan. This may be because these tumors were flat and plate-like in shape, free-floating and mobile in the vasculature. Another possibility is that the tumor grew rapidly in the 5 days between the $\mathrm{CT}$ scan and the operation. However, given that $\mathrm{CT}$ at a previous hospital performed 3 weeks earlier indicated similar findings, it appears reasonable to conclude that the sensitivity of CT was limited for the detection of free-floating lesions in the bloodstream. In fact, the sensitivity of CT angiography for detection of a free-floating thrombus has been reported to be only $60 \%$ (14). Multislice CT may exhibit a better efficacy (15), although this depends on the clinical conditions and the form of the intravascular tumor. As regards the present case, it was crucial to confirm the location of the upper tip of the tumor by perioperative ultrasound scanning, regardless of the CT findings. Otherwise, instant transection of the IVC may have caused pulmonary embolism. The key educational point of this case is that, although CT is widely performed, it is important to acknowledge the limitations of this method. 
It was previously reported that $>50 \%$ of intravenous LG-ESS cases exhibited ICE (8). In such cases, the extent of the tumor should be determined using modalities such as MRI and ultrasonography, in addition to CT. MRI provides good soft tissue resolution and helps to distinguish an intravascular tumor from a non-tumor thrombus (16-18), whereas Doppler ultrasonography is also useful (16). Transthoracic ultrasonography of the IVC and heart would normally be performed. However, the transesophageal method may be selected in a case of unknown tumor origin or in which the tumor extent is unclear by transthoracic examination, or when detailed knowledge of the cardiac function is required, such as cases with tricuspid valve involvement (19). Indeed, perioperative transesophageal echocardiography was required in our patient.

Our case was asymptomatic and was incidentally discovered during a routine medical checkup. This was rather fortunate, as fatal congestive heart failure may be the initial presentation $(8,20)$. In this case, elevation of serum liver enzymes was most likely caused by another reason, since it persisted even after tumor removal. However, liver injury may be caused by liver congestion due to the intravascular tumor in the IVC and right atrium in similar cases; therefore, transesophageal echocardiography may be useful prior to surgery. 18-Fluorodeoxyglucose (FDG) positron emission tomography-CT (PET-CT) may also be a viable choice for determining the tumor location or distinguishing between sarcoma and benign leiomyoma. PET-CT has been reported as an effective tool for the detection of uterine sarcoma metastasis (21), but reports are limited for LG-ESS $(22,23)$. Certain tumors and metastatic lesions are known to exhibit a low FDG uptake (24) and its accuracy for intravascular lesions is unreported to date; thus, even if performed preoperatively, tumors in the IVC above the level of the renal veins may not be detected.

Multimodal imaging is costly and time-consuming, but it is important to determine the extent of a tumor preoperatively, as it significantly affects surgical resection planning, including route, order of procedures, methods, devices and the need for extracorporeal circulation.

We herein presented a case of LG-ESS with ICE, in which the extent of the tumor was not accurately predicted on preoperative CT. In similar cases, accurate determination of the tumor extent for planning the surgical strategy should be performed using multiple imaging methods.

\section{Acknowledgements}

This manuscript was proofread by a native English-speaking proofreader of the Palabra Language Services, Kyoto, Japan.

\section{References}

1. Brooks SE, Zhan M, Cote T and Baquet CR: Surveillance, epidemiology and end results analysis of 2677 cases of uterine sarcoma 1989-1999. Gynecol Oncol 93: 204-208, 2004.

2. Sagae S, Yamashita K, Ishioka S, Nishioka Y, Terasawa K, Mori M, Yamashiro K, Kanemoto T and Kudo R: Preoperative diagnosis and treatment results in 106 patients with uterine sarcoma in Hokkaido, Japan. Oncology 67: 33-39, 2004

3. Fujita H, Adachi S, Kigawa J, Sugiyama T and Takeuchi S: A clinicopathological study of uterine sarcoma in last decade - a retrospective study of KCOG/USSG inter group study. Adv Obstet Gynecol 56: 463-465, 2004 (In Japanese).
4. Hendrickson MR, Tavassoli FA, Kempson RL, et al: Mesenchymal tumors and related lesions. In: Pathology and Genetics of Tumours of the Breast and Female Genital Organs. Tavassoli FA and Devilee P (eds). 3rd edition. International Agency for Research on Cancer, Lyon, pp233-244, 2003.

5. Oliva E, Carcangiu ML, Carinelli SG, et al: Mesenchymal tumors. In: WHO Classification of Tumours of Female Reproductive Organs. 4th edition. Kurman RJ, Carcangiu ML, Herrington CS and Young RH (eds). International Agency for Research on Cancer, Lyon, pp135-147, 2014.

6. Leath CA III, Huh WK, Hyde J Jr, Cohn DE, Resnick KE, Taylor NP, Powell MA, Mutch DG, Bradley WH, Geller MA, et al: A multi-institutional review of outcomes of endometrial stromal sarcoma. Gynecol Oncol 105: 630-634, 2007.

7. Riopel J, Plante M, Renaud MC, Roy M and Têtu B: Lymph node metastases in low-grade endometrial stromal sarcoma. Gynecol Oncol 96: 402-406, 2005.

8. Lo KW, Yu MY and Cheung TH: Low-grade endometrial stromal sarcoma with florid intravenous component. Gynecol Obstet Invest 66: 8-11, 2008.

9. Gabal S, Ashour Z, Hamada G, Aziz SA, Khairy H, Badawy H, Hamada EM and Saied K: Low-grade endometrial stromal sarcoma with intravenous extension to the heart. Medscape J Med 11: 23, 2009.

10. Dzieciuchowicz ŁS, Słowinski M, Brzeziński JJ and Kycler W: Tumor embolus due to uterine cancer. Med Sci Monit 15: CS155-CS157, 2009.

11. Nagumo M, Kiso I, Misumi T, Yasudo M, Nakada K and Mukai M: Cardiac extension of intravenous leiomyomatosis with successful resection. Tokai J Exp Clin Med 22: 125-131, 1997.

12. Tamura R, Kashima K, Asatani M, Nishino K, Nishikawa N, Sekine M, Serikawa T and Enomoto T: Preoperative ultrasound-guided needle biopsy of 63 uterine tumors having high signal intensity upon T2-weighted magnetic resonance imaging. Int J Gynecol Cancer 24: 1042-1047, 2014.

13. Ueda M, Otsuka M, Hatakenaka M and Torii Y: Uterine endometrial stromal sarcoma located in uterine myometrium: MRI appearance. Eur Radiol 10: 780-782, 2000.

14. Ferrero E, Ferri M, Viazzo A, Labate C, Pecchio A, Berardi G, Piazza S, Cumbo P and Nessi F: Free-floating thrombus in the internal carotid artery: Diagnosis and treatment of 16 cases in a single center. Ann Vasc Surg 25: 805-812, 2011.

15. Sun C, Wang XM, Liu C, Xv ZD, Wang DP, Sun XL and Deng K: Intravenous leiomyomatosis: Diagnosis and follow-up with multislice computed tomography. Am J Surg 200: e41-e43, 2010.

16. Fasih N, Prasad Shanbhogue AK, Macdonald DB, Fraser-Hill MA, Papadatos D, Kielar AZ, Doherty GP, Walsh C, McInnes M and Atri M: Leiomyomas beyond the uterus: Unusual locations, rare manifestations. Radiographics 28: 1931-1948, 2008.

17. Cohen DT, Oliva E, Hahn PF, Fuller AF Jr and Lee SI: Uterine smooth-muscle tumors with unusual growth patterns: Imaging with pathologic correlation. AJR Am J Roentgenol 188: 246-255, 2007.

18. Kang LQ, Zhang B, Liu BG and Liu FH: Diagnosis of intravenous leiomyomatosis extending to heart with emphasis on magnetic resonance imaging. Chin Med J (Engl) 125: 33-37, 2012.

19. Baca López FM, Martínez-Enriquez A, Castrejón-Aivar FJ, Ruanova-León D and Yánez-Gutiérrez L: Echocardiographic study of an intravenous leiomyoma: Case report and review of the literature. Echocardiography 20: 723-725, 2003.

20. Barksdale J, Abolhoda A and Saremi F: Intravenous leiomyomatosis presenting as acute Budd-Chiari syndrome. J Vasc Surg 54: 860-863, 2011.

21. Sadeghi R, Zakavi SR, Hasanzadeh M, Treglia G, Giovanella L and Kadkhodayan S: Diagnostic performance of fluorine-18-fluorodeoxyglucose positron emission tomography imaging in uterine sarcomas: Systematic review and meta-analysis of the literature. Int J Gynecol Cancer 23: 1349-1356, 2013.

22. Luo Y, Feng R and Li F: FDG PET/CT appearance of tumor thrombus of ovarian vessels masquerading as retroperitoneal fibrosis. Clin Nucl Med 40: 501-503, 2015.

23. Takizawa M, Tanaka N, Tsunezuka Y, Katayanagi K and Kurumaya H: Solitary pulmonary metastasis of low-grade uterine endometrial stromal sarcoma resected 31 years before. Japan J Thorac Surg 67: 333-336, 2014 (In Japanese).

24. Inoue $\mathrm{K}$, Tsubamoto $\mathrm{H}$, Kawata S, Hao H, Ikeda $\mathrm{Y}, \mathrm{Oku} \mathrm{N}$ and Hirota S: ${ }^{18}$ F-fluorodeoxyglucose uptake and clinicopathological features of recurrent or metastatic endometrial stromal sarcoma. J Obstet Gynaecol Res 40: 576-582, 2014 